\title{
DEVELOPMENT OF METHOD TO EVALUATE FRICTION LOSSES OF CHAIN DRIVES
}

\author{
Konstantin Kozlov, Vladimir Belogusev, Aleksey Egorov, Nikolay Syutov \\ Volga State University of Technology, Russia \\ konstantin.k-e@yandex.ru, vladimir.belogusev@yandex.ru, al.v.egorov@yandex.ru
}

\begin{abstract}
The power of mechanical losses in chain drives is an important characteristic of the quality of their manufacture, since the internal resistances in the bearing assemblies, as well as in the chain hinges, not only influence the energy efficiency of the mechanical system, but also have a significant effect on the rate of degradation processes, and hence its durability and reliability. Widely used methods and instruments for control of mechanical losses in chain drives, for example, strain measurement, do not allow identifying the required parameters with sufficient accuracy in the dynamic modes of operation, and very often require expensive measuring equipment. The purpose of the present paper is to develop a method and instruments for highprecision control of the quality of assembling and manufacturing of chain drives based on identifying the energy loss in their friction units in dynamic modes of operation. This study presents the results of theoretical and experimental substantiation of the method and instruments being developed. The accuracy of the control of the mechanical parameters of chain drives, and, consequently, the quality of their control when using the presented method, is increased by several times in comparison with the results obtained by other existing methods. The developed method can be used for accelerated test of chain drives and chains at manufacturing companies. Having determined the acceptable level of losses in the chain drives, it can be possible to build a system for assessing their technical state, determine their efficiency, reliability and durability with minimal financial and time costs. In further research, we plan to improve the design of the test bench for easy replacement and installation of chain drives in order to reduce the laboriousness of the test process based on the developed method.
\end{abstract}

Keywords: chain performance, chain drive, chain efficiency, retardation method, free running.

\section{Introduction}

The power of mechanical losses in chain drives is an important indicator of the quality of its manufacture. Internal friction in bearing units, as well as those that arise during the rotation of chain hinges, not only influence the energy efficiency of the mechanical system, but also have a significant effect on the rate of degradation processes, and, consequently, the durability and reliability of a chain drive [1]. Therefore, studies aimed at creating high-precision methods for measuring their performance are relevant.

A high value of the chain drive efficiency requires the use of precise control methods, however, at present, the efficiency is determined either by calculation $[2 ; 3]$ or experimentally [4-8]. Calculation methods use a huge number of assumptions, which limits their application. Among the experimental methods for determining the mechanical efficiency of a rotating system, the tensometric method is most widely used, which requires high accuracy of signal measurement and calibration of strain sensors $[4 ; 5 ; 7 ; 9]$. In this case, the determination of energy losses performs with a relatively large discreteness, which is associated with the time needed to restore the deformed state of the strain element. It is also limited by the complexity of removing the signal from the strain elements, as well as the high cost of the measuring equipment $[10 ; 11]$.

Also the method presented in [1] is known, which uses the acceleration characteristics of asynchronous electric drive to determine the efficiency of chain drives. However, it should be noted that these studies do not take into account the errors associated with the instability of the voltage applied to the induction motor, which is in quadratic dependence on the external mechanical characteristic of it $[12 ; 13]$. In addition, the use of rotary bodies of different weights with the known moments of inertia does not allow the creation of equal frictional conditions in the bearing units in the test bench. The listed drawbacks lead to significant errors in testing, which limits their application to high efficiency drives. On the basis of the analysis it can be concluded that the existing methods for control of the quality of chain drives do not allow evaluating their performance with sufficient accuracy.

The purpose of this paper is to develop a method for evaluation of the quality of chain drives, which has high measurement accuracy in a wide range of speeds. 


\section{Materials and methods}

\section{A method to evaluate the quality of a chain drive}

The method proposed in this paper is based on measuring the level of mechanical losses in the chain drive in a wide range of speeds during the retardation of the test bench, which includes a chain drive (see Fig. 1). In this case, the evaluation criterion is the braking torque produced in the system by frictional forces in all units of the mechanical system.

Fig. 1 shows a scheme of a test bench.

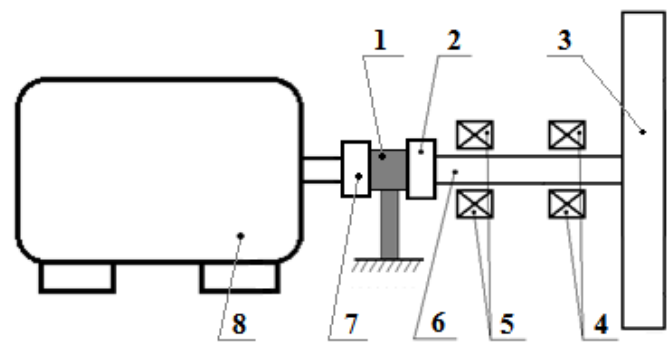

(a)

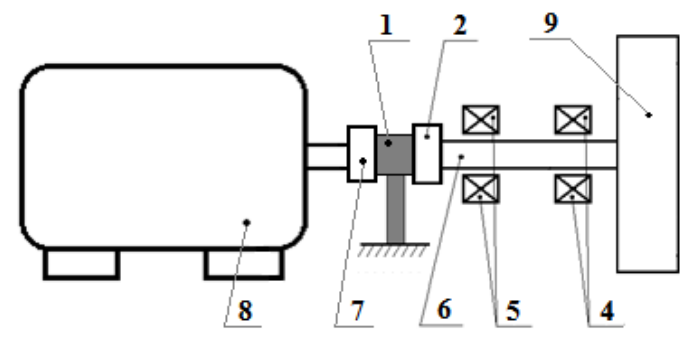

(b)

Fig. 1. Scheme of test bench: with a rotary body having the weight $m$ and the moment of inertia $J_{1}(a)$; with a rotary body having the weight $m$ and the moment of inertia $J_{2}(b): 1$ - encoder; 2 - coupling for an encoder; 3 and 9 - reference disks, having the identical weights but different known values of the moment of inertia; 4 and 5 - rolling bearings; 6 - shaft; 7 - electromagnetic coupling; 8 - drive motor

At the first stage, we determine the braking torque in the rotary system "encoder 1 , shaft 6 , coupling 2 , coupling 7, rolling bearings 4 and 5", which is caused by mechanical losses in the friction units.

After mounting the rotary body 3 on the shaft 6 (Fig. 1a), we accelerate the rotating system by help of the drive motor 8 . When the rated speed of rotation is reached, we disconnect the drive motor, and by help of the encoder 1 , we register the values of the angular acceleration $\varepsilon_{1}$ at retardation from the rated speed of rotation to zero one. In this case, the braking torque is calculated as follows:

$$
M_{B T B}=\left(J_{1}+J_{T B}\right) \cdot \varepsilon_{1}=\left(J_{\Delta}+J_{2}+J_{T B}\right) \cdot \varepsilon_{1},
$$

where $J_{1}$ - moment of inertia of a big rotary body of the weight $\mathrm{m}, \mathrm{kg} \cdot \mathrm{m}^{2}$;

$J_{2}$ - moment of inertia of a small rotary body of the weight $m, \mathrm{~kg} \cdot \mathrm{m}^{2}$;

$J_{B T B}-$ moment of inertia of the test bench without a rotary body, $\mathrm{kg} \cdot \mathrm{m}^{2}$;

$J_{\Delta}$ - difference between the values of the moments of inertia $J_{1}$ and $J_{2}, \mathrm{~kg} \cdot \mathrm{m}^{2}$.

Then, we substitute the rotary body 3 for the rotary body 9 (Fig. 1, b). In this case, the moment of inertia of the rotating system decreases, and, consequently, the resultant angular acceleration during the retardation from the rated speed of rotation to zero one increases (since the amount of energy that a rotary body with a smaller moment of inertia can accumulate decreases). In this case, the weight of the rotary body 9 does not change, and, consequently, the level of mechanical losses in the bearing units also remains constant. By help of the drive motor, the rotating system accelerates, and the angular velocity is brought to the rated one. Then we disconnect the drive motor and register the resultant angular acceleration $\varepsilon_{2}$ during self-braking. 
In this case, the braking torque is calculated as follows:

$$
M_{B T B}=\left(J_{2}+J_{T B}\right) \cdot \varepsilon_{2}
$$

Since two processes of retardation (with rotary body 3 and rotary body 9) are performed with identical friction level in the rotary units, therefore, we can equate the right-hand parts of Eq. 1 and Eq. 2 and find $J_{T B}+J_{2}$ as follows:

$$
J_{T B}+J_{2}=J_{\Delta} \cdot \frac{\varepsilon_{1}}{\varepsilon_{2}-\varepsilon_{1}} .
$$

The braking torque $M_{T B T}$ is calculated as follows:

$$
M_{T B T}=J_{\Delta} \cdot \frac{\varepsilon_{1} \cdot \varepsilon_{2}}{\varepsilon_{2}-\varepsilon_{1}} .
$$

To determine the energy losses in a chain drive, we use the test bench described above and mount the chain drive 10 to its shaft 6 (Fig. 2).

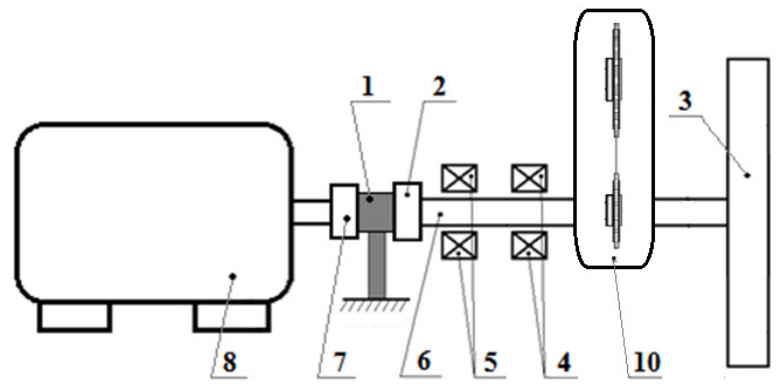

(a)

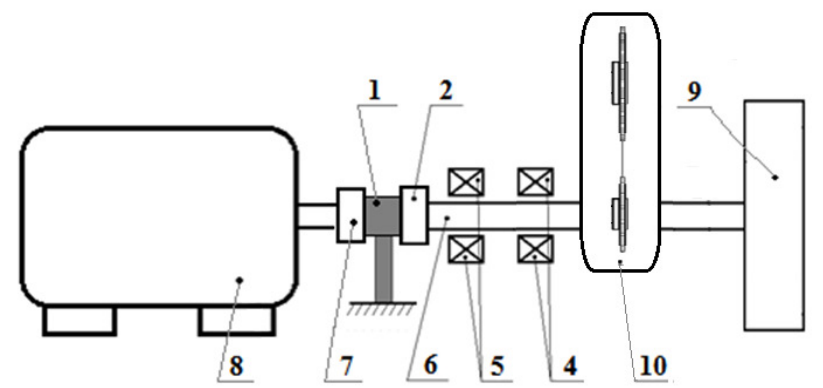

(b)

Fig. 2. Scheme of test bench with chain drive with a rotary body of the weight $m$ and the moment of inertia $J_{1}(a)$; with a rotary body of the weight $m$ and the moment of inertia $J_{2}(b)$

Similarly, as in the case of the scheme in Fig. 1, after the acceleration of the rotating system with the rotary body of the moment of inertia $J_{1}$, by help of the encoder 1 , we register the values of the angular acceleration $\varepsilon_{3}$ during the retardation of the considered rotating system from the rated speed of rotation to the zero one. In this case, the braking torque of mechanical losses can be calculated as follows:

$$
M_{B T B C T}=\left(J_{1}+J_{T B+C T}\right) \cdot \varepsilon_{3}=\left(J_{\Delta}+J_{2}+J_{T B+C T}\right) \cdot \varepsilon_{3},
$$

where $J_{T B+C T}-$ moment of inertia of the rotating system of a test bench with a chain drive without a rotary body, $\mathrm{kg} \cdot \mathrm{m}^{2}$;

$J_{\Delta}$ - difference between the values of the moments of inertia $J_{1}$ and $J_{2}, \mathrm{~kg} \cdot \mathrm{m}^{2}$.

Then we substitute the body 3 for the rotary body 9 and determine the resultant angular acceleration $\varepsilon_{4}$ of the rotating system with the chain drive and the rotary body 9 .

In this case, the braking torque can be calculated as follows:

$$
M_{B T B C T}=\left(J_{2}+J_{T B+C T}\right) \cdot \varepsilon_{4} \text {. }
$$


Since two processes of retardation (with rotary body 3 and rotary body 9) are performed with identical friction level in the rotary units, therefore, we can equate the right-hand sides of Eq. 5 and Eq. 6 and find the value of $J_{T B+C T}+J_{2}$ :

$$
J_{T B+C T}+J_{2}=J_{\Delta} \cdot \frac{\varepsilon_{3}}{\varepsilon_{4}-\varepsilon_{3}} . \text { (7) }
$$

The braking torque of the rotating system "test bench, chain drive" can be calculated as follows:

$$
M_{B T B C T}=J_{\Delta} \cdot \frac{\varepsilon_{3} \cdot \varepsilon_{4}}{\varepsilon_{4}-\varepsilon_{3}} .
$$

The braking torque caused by mechanical losses in the chain drive can be calculated as follows:

$$
M_{B C T}=M_{B T B C T}-M_{B T B} .
$$

Instruments to evaluate the quality of a chain drive

For experimental substantiation of the developed theory a test bench was assembled on the basis of an asynchronous electric motor (Fig. 3). The test bench allows measuring the acceleration of the rotating system and processing the data obtained. In this case, an asynchronous electric motor is possible to easily detach from the test bench during rotation.

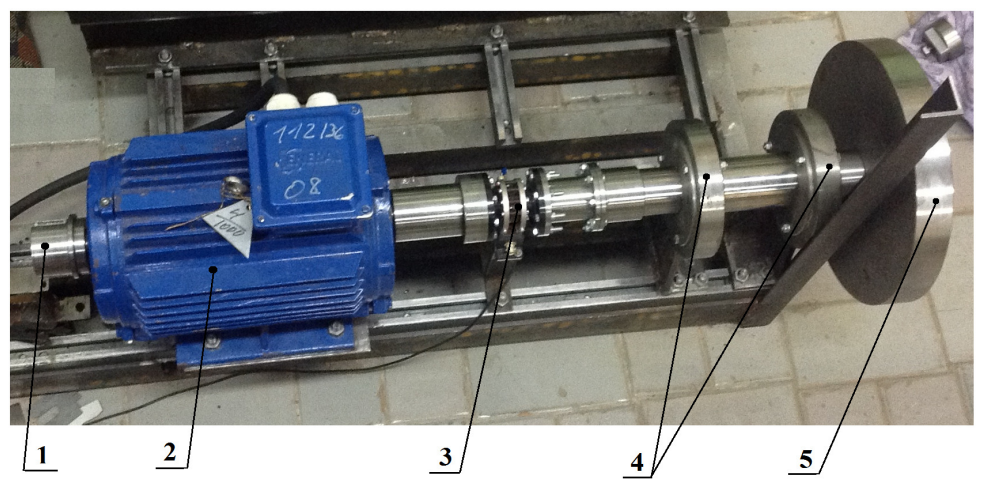

Fig. 3. Test bench for experimental substantiation of developed method for evaluating quality of chain drive: 1 - seat for fixing a rotary body with the known moment of inertia; 2 - induction motor AIR 112MV6 (220/380 V), 4 kW, $n=940 \mathrm{rpm} ; 3$ - encoder E100H; 4 - bearing units; 5 - rotary body with the known moment of inertia

To determine the angular accelerations in the experiments, the incremental encoder E100H manufactured by Autonics was used, which produces 512 pulses per revolution. For its placement between the motor shaft and the shaft with bearing units 4, a special coupling was made.

Fig. 4 shows a scheme of the developed hardware and software complex for recording and processing signals incoming from the encoder. The signal goes to the input of the registrating unit. The measuring microcontroller measures the duration of the signal with the microcontroller clock frequency. The used CPU has a frequency of $16 \mathrm{MHz}$, which provides a negligibly small error in recording the time needed to rotate the measuring disk by the value of angle between two closest graduation marks on it at the angular velocity of the rotating system of up to $940 \mathrm{rpm}$.

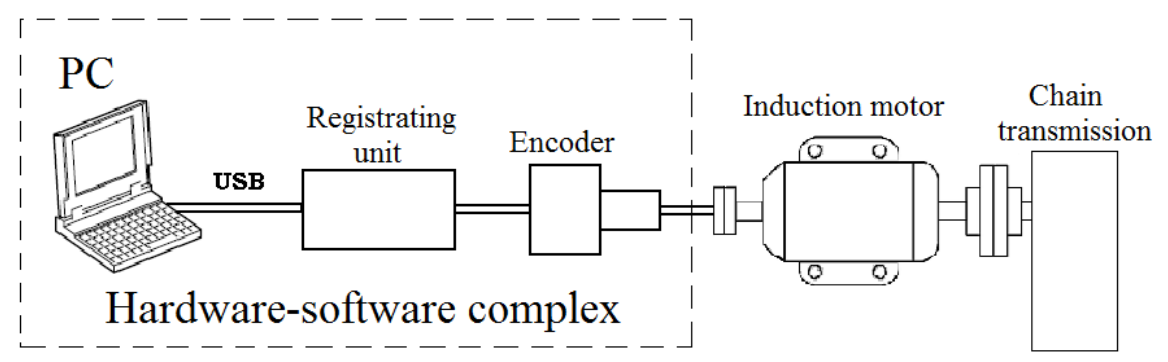

Fig. 4. Scheme to control friction losses in chain drive 
An array of values is transmitted via the UART interface to the galvanic isolation system. Then the data array enters the personal computer (PC) and is recorded using the terminal program. In the terminal program on a $\mathrm{PC}$, the data array is mathematically processed and the angular velocities and accelerations of the rotating system under study are calculated.

For statistical processing of the experimental data, the technique of determining the minimum required number of measurements using the Student's t-distribution was used [14]. As a result of the experiments and calculations according to this technique, for the determination of the moment of inertia at the accuracy of $0.5 \%$, there were performed two series of experiments, consisting of 10 measurements of the angular acceleration each.

\section{Results and discussion}

Figures 5 and 6 describe an experiment based on the measurements of the angular velocity of a rotating system.

To substantiate the increase in the accuracy of measuring the power of energy losses in friction units using the proposed method, we used an additional rotary body 11 with the known moment of inertia. In this experiment the induction motor AIR 112MV6 $(220 / 380 \mathrm{~V}), 4 \mathrm{~kW}, n=940 \mathrm{rpm}$ was used. As rotary bodies 3 and 9, ones of the identical weights and with the moments of inertia of $0.2579 \mathrm{~kg} \cdot \mathrm{m}^{2}$ and $0.45127 \mathrm{~kg} \cdot \mathrm{m}^{2}$ were used.

The moment of inertia of the rotary body 11 was measured by two methods (existing one (Fig. 5) and developed one (Fig. 6)).

In the application of both methods we determined the minimum required number of measurements needed to satisfy the accuracy of $0.5 \%$, and, as a result, 10 measurements were carried out, blunders were eliminated, the average moment of inertia values were found, and the relative random error values, which were in the range of 0.259-0.389\%, were determined.

According to the existing method developed in [1], we determined the desired value for the entire rotating system shown in Fig. 5 without the rotary body 11. Then, we determined one for the entire rotating system, represented in Fig. 5 with the rotary body 11. The difference between two moments of inertia was compared with the true value of the moment of inertia of the rotary body 11 .

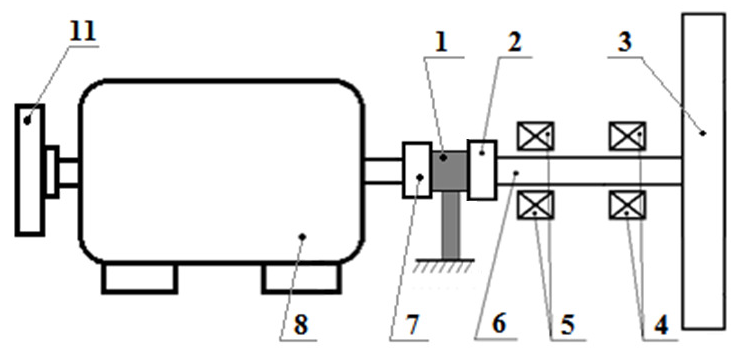

Fig. 5. Scheme of experimental determination of moment of inertia of additional rotary body by existing method: 1 - encoder; 2 - half-coupling; 3 - rotary body with the known moment of inertia; 4, 5 - bearings; 6 - shaft; 7 - half-coupling; 8 -induction motor; 11 - rotary body, the moment of inertia of which was determined during the experiment

The results are shown in Table 1.

Table 1

Inaccuracy in measuring the moment of inertia of the rotary body 11 using the existing method

\begin{tabular}{|c|c|c|c|c|c|c|}
\hline $\boldsymbol{n}, \mathbf{r p m}$ & $\mathbf{2 0 0 - 3 0 0}$ & $\mathbf{3 0 0 - 4 0 0}$ & $\mathbf{4 0 0 - 5 0 0}$ & $\mathbf{5 0 0 - 6 0 0}$ & $\mathbf{6 0 0 - 7 0 0}$ & $\mathbf{7 0 0 - 8 0 0}$ \\
\hline $\boldsymbol{J}_{\text {true }}, \mathbf{k g} \cdot \mathbf{m}^{\mathbf{2}}$ & 1.797 & 1.797 & 1.797 & 1.797 & 1.797 & 1.797 \\
\hline $\boldsymbol{J}_{\text {deter }}, \mathbf{k g} \cdot \mathbf{m}^{2}$ & 1.709 & 1.725 & 1.695 & 1.629 & 1.699 & 1.679 \\
\hline$\Delta \mathbf{~ k g} \cdot \mathbf{m}^{2}$ & 0.088 & 0.072 & 0.102 & 0.168 & 0.098 & 0.118 \\
\hline $\boldsymbol{\delta}, \mathbf{\%}$ & 4.897 & 4.007 & 5.676 & 9.349 & 5.454 & 6.567 \\
\hline
\end{tabular}

In the next step, we determined the moment of inertia of the rotary body 11 according to the scheme shown in Fig. 6 using the developed in this paper method: we determined the moment of inertia of the entire rotating system shown in Fig. 6 without the rotary body 11; then we determined 
the moment of inertia of the entire rotating system with the rotary body 10; the difference between two moments of inertia was compared with the true value.

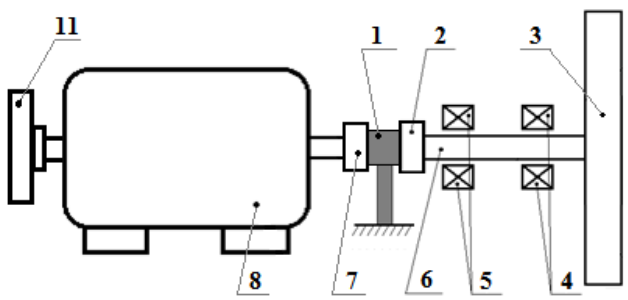

(a)

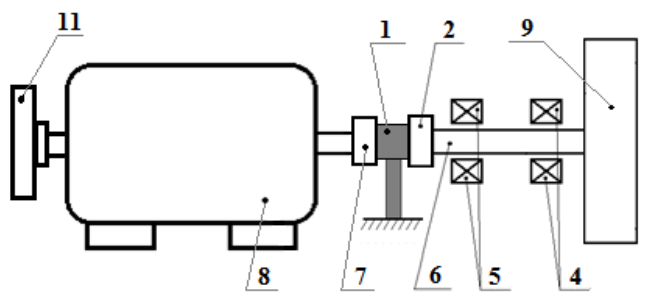

(b)

Fig. 6. Scheme for experimental determination of moment of inertia of rotary body by developed method with a rotary body of the weight $m$ and the moment of inertia $J_{1}(a)$; with a rotary body of the weight $\boldsymbol{m}$ and the moment of inertia $\boldsymbol{J}_{\mathbf{2}}$ (b): 1 - encoder; 2 - half-coupling; 3 and 9 - rotary bodies with the same weight but with different known moments of inertia; 4 and 5 - bearings; 6 - shaft; 7 - half-coupling; 8 - induction motor; 11 - rotary body, the moment of inertia of which was determined during the experiment.

Table 2 shows the results obtained.

Inaccuracy of measuring the moment of inertia of the rotary body 11 with the help of the developed method

\begin{tabular}{|c|c|c|c|c|c|c|}
\hline $\boldsymbol{n}, \mathbf{r p m}$ & $\mathbf{2 0 0 - 3 0 0}$ & $\mathbf{3 0 0 - 4 0 0}$ & $\mathbf{4 0 0 - 5 0 0}$ & $\mathbf{5 0 0 - 6 0 0}$ & $\mathbf{6 0 0 - 7 0 0}$ & $\mathbf{7 0 0 - 8 0 0}$ \\
\hline $\boldsymbol{J}_{\text {true }}, \mathbf{k g} \cdot \mathbf{m}^{\mathbf{2}}$ & 1.797 & 1.797 & 1.797 & 1.797 & 1.797 & 1.797 \\
\hline $\boldsymbol{J}_{\text {deter }}, \mathbf{~} \mathbf{~ k g} \cdot \mathbf{m}^{\mathbf{2}}$ & 1.765 & 1.793 & 1.805 & 1.799 & 1.825 & 1.792 \\
\hline$\Delta \mathbf{~} \mathbf{g} \cdot \mathbf{m}^{\mathbf{2}}$ & 0.032 & 0.004 & -0.008 & -0.002 & -0.028 & 0.005 \\
\hline $\boldsymbol{\delta}, \boldsymbol{\%}$ & 1.781 & 0.223 & -0.445 & -0.111 & -1.558 & 0.278 \\
\hline
\end{tabular}

On the basis of the results obtained in Table 1 and Table 2, it can be concluded that the accuracy of measuring the moment of inertia and, consequently, the power of mechanical losses in the chain drive, when using the developed method, has increased almost 4 times in comparison with the measurement accuracy of the existing method. The obtained values confirm the theoretical assumptions based on the possibility of increasing the accuracy of the control of friction losses in the parts mating of the chain drive thanks to the use of bodies of rotation of the same weight values, but different values of the moments of inertia. In addition, a significant difference in the results was also achieved owing to the absence of influence of supply voltage deviations that occurred in the first experiment (Fig. 5), since the method developed in this paper (Fig. 6) is based on the retardation time of the rotating elements system, and not on the time of its acceleration by help of an asynchronous electric motor. Hypothetically, it will be also possible to increase the accuracy of measurements, when the electric motor is disconnected from the system of rotating elements of a chain drive after acceleration, which will ensure that there will be no effect of the remanent magnetization of an electric motor.

According to the data obtained in the experiments, it can also be concluded that the highest measurement accuracy is achieved in the speed range of 300-600 rpm. Most likely, this is due to uncontrolled processes of mains supply influence and remanent magnetization of the stator windings in the first seconds of the retardation of the rotating elements system, since in the experiments 
conducted, the electric motor was not detached from the system under investigation after it was accelerated. In further studies, it is planned to verify the validity of this hypothesis.

\section{Conclusions}

According to the results obtained, the developed method makes it possible to improve the accuracy of determining the efficiency of chain drives, and, consequently, the quality of their control.

This method can be used for accelerated tests at manufacturers of chain drives and chains to determine their efficiency, durability and reliability. Having determined the permissible level of losses in the friction units, it is possible to build a system for assessing their technical condition.

In the future, it is planned to develop a design for easy replacement and installation of chain drives in order to reduce the laboriousness of the measurement process using the developed method.

\section{References}

[1] Egorov A., Kozlov K., Belogusev V. A Method for evaluation of the chain drive efficiency. Journal of Applied Engineering Science, vol. 4(13), 2015, pp. 277-282.

[2] Liu S.P., Wang K.W., Hayek S.I. Modeling and analysis of chain drive systems. The Journal of the Acoustical Society of America, vol. 87, 1990, pp. 136-139.

[3] Zhang L., Zhang C., Horng J.-H.etc. Study on Simulation of the Chain Transmission Mechanism. Advanced Materials Research, vol. 593, 2012, pp. 797-800.

[4] Conwell J.C, Johnson G.E. Design, construction and instrumentation of a machine to measure tension and impact forces in roller chain drives. Mechanism and Machine Theory, vol. 31, 1996, pp. 525-531.

[5] Conwell J.C., Johnson G.E. Experimental investigation of link tension and roller sprocket impact forces in roller chain drives. Mechanism and Machine Theory, vol. 31, 1996, pp. 533-544.

[6] Doppelbauer M. Accuracy of the Determination Of Losses and Energy Efficiency of Induction Motors by the Indirect Test Procedure, EEMODS, 2011, Washington, USA.

[7] Irimescu A., Mihon L., Pädure G. Automotive transmission efficiency measurement using a chassis dynamometer. International Journal of Automotive Technology, vol. 12, 2011, pp. 555559.

[8] Huo J., Yu S., Yang J. etc. Static and Dynamic Characteristics of the Chain Drive System of a Heavy Duty Apron Feeder. The Open Mechanical Engineering Journal, vol. 7, 2013, pp. 121-128.

[9] Troedsson I., Vedmar L.A Method to Determine the Static Load Distribution in a Chain Drive. ASME Journal of Mechanical Design, vol. 121, 1999, pp. 402-408.

[10] Spicer J.B., Richardson C.J.K., Ehrlich M.J. etc. Effects of Frictional Loss on Bicycle Chain Drive Efficiency. Journal of Mechanical Design, vol. 123, 1999, pp. 598-605.

[11] Srivastava N., Haque I. A review on belt and chain continuously variable transmissions (CVT): Dynamics and control. Mechanism and Machine Theory, vol. 44, 2009, pp. 19-41.

[12] Bhattacharya S.K. Electrical machines. Third edition. New Delhi: Tata McGraw-Hill Education, 2009. $624 \mathrm{p}$.

[13] Pyrhonen J., Jokinen T., Hrabovcova V. Design of rotating electrical machines. First edition. Chippenham: John Wiley \& Sons, 2008. 538 p.

[14] Krutov V.I., Grushko I.M., Popov V.V. etc. Fundamentals of Scientific Research (Основы научных исследований). Moscow: «Высшая школа», 1989. 400 p. (In Russian). 